\title{
Analysis of Methanol Maser Flares in G107.298+5.63 and S255-NIRS3
}

\author{
M. D. Gray ${ }^{1,2}$, S. Etoka ${ }^{1}$ and B. Pimpanuwat ${ }^{1,2}$ \\ ${ }^{1}$ Jodrell Bank Centre for Astrophysics, Department of Physics and Astronomy, University of Manchester, M13 9PL, UK \\ ${ }^{2}$ National Astronomical Research Institute of Thailand, 260 Moo 4, T. Donkaew, A. Maerim, Chiangmai 50180, Thailand.
}

\begin{abstract}
A 3D maser model has been used to perform an inverse problem on the light curves from three high-amplitude maser flares, selected on the basis of contemporaneous infra-red observations. Plots derived from the model recover the size of the maser cloud, and two parameters linked to saturation, from three observational properties of the light curve. Recovered sizes are consistent with independent interferometric measurements. Maser objects transition between weak and moderate saturation during a flare.
\end{abstract}

Key words: masers - radiative transfer - radio lines: general - radiation mechanisms: general - techniques: high angular resolution - ISM: lines and bands.

\section{INTRODUCTION}

In a recent paper (Gray et al. 2020) (Paper 1), we demonstrated that radiatively-driven maser flares may be usefully characterised by three statistics derived from their light curves: the variability index, the duty cycle, and the maximum flux density achieved during the flare. The variability index is defined here as the maximum flux density divided by the minimum, if the source is periodic, or the maximum flux density divided by the pre-flare quiescent level for ' an aperiodic source. The duty cycle is defined as the fraction of a characteristic time for which the maser flux density is above half the peak value; the characteristic time is the flare period for periodic sources, and the time for which the flare is considered active for aperiodic sources.

The duty cycle is a particularly useful statistic because it provides a clear distinction between flares that are driven by variation in the pumping radiation and flares that are driven by variations in the background radiation. Maser flares of the former type rarely have a duty cycle that exceeds that of the driving function, that is the light curve of the infra-red (IR) pumping radiation, whilst the latter type almost invariably have a duty cycle greater than that of the driving function of the radio background, at least for flares of significant variability index $(>1.5)$.

In the present work, guided by observational constraints on the duty cycle and variability index, we provide fits to spectral components with flares in two star-forming region sources: G107.298+5.63 and S255-NIRS3. The former source exhibits periodic flares in the $6.7-\mathrm{GHz}$ maser transition of methanol, whilst the latter source is an example of an accretion burst source that is aperiodic with extreme peak flux densities $(>1000 \mathrm{Jy})$ in some spectral components. The period of the methanol masers in G107.298+5.63 is $34.6 \mathrm{~d}$, an interval that applies also to $22-\mathrm{GHz} \mathrm{H}_{2} \mathrm{O}$ masers in the source (Szymczak et al. 2016). Perhaps the most plausible physical model for the overall G107.298+5.63 flare mechanism is a colliding-wind binary (Stecklum et al. 2018). G107.298+5.63 and S255-NIRS3 were chosen because they are examples of a currently very select group that have IR light curves measured contemporaneously with the maser data. In G107.298+5.63, we consider the spectral component at $-7.4 \mathrm{~km} \mathrm{~s}^{-1}$ and in S255-NIRS3, the components at 6.42 and $5.83 \mathrm{~km} \mathrm{~s}^{-1}$ (see Fig. 3 of Uchivama et al. 2020).

\section{MODEL AND OBSERVATIONAL DATA}

The model used here is as described in Paper 1, and is based on earlier versions developed in Gray et al. (2018) and Gray et al. (2019). Data used in Paper 1 was supplemented for the present work by a series of models with a shell-like distribution of the unsaturated inversion with a distribution $\propto r^{2}$, where $r$ is the radial distance from the domain centre. Data from the model with the shell distribution were used to estimate modelling uncertainties. All model domains were prolate objects with a distortion factor of $\Gamma=0.6$ (see Gray et al. (2019) for definition), and viewed from the optimum direction. This combination of source shape and viewing direction were found to give the largest flux densities for a source of given volume and mean unsaturated inversion. Viewing from a random orientation reduces the peak flux density for this type of object by approximately two orders of magnitude (see Fig. 15 of Paper 1).

A convenient way to present data from a large number of models is to present one statistic, the maximum flux density, as a colour palette, and the other two statistics via sets of contours, all on the same set of axes. These axes show the initial optical depth parameter of the model on the $x$ axis, noting that the depth parameter does not change in variable background models, and the change in the driving function on the $y$ axis. A single point in the $x y$-plane of a diagram of this type involves computing an entire maser light curve, even though only the three statistics derived from it are plot- 


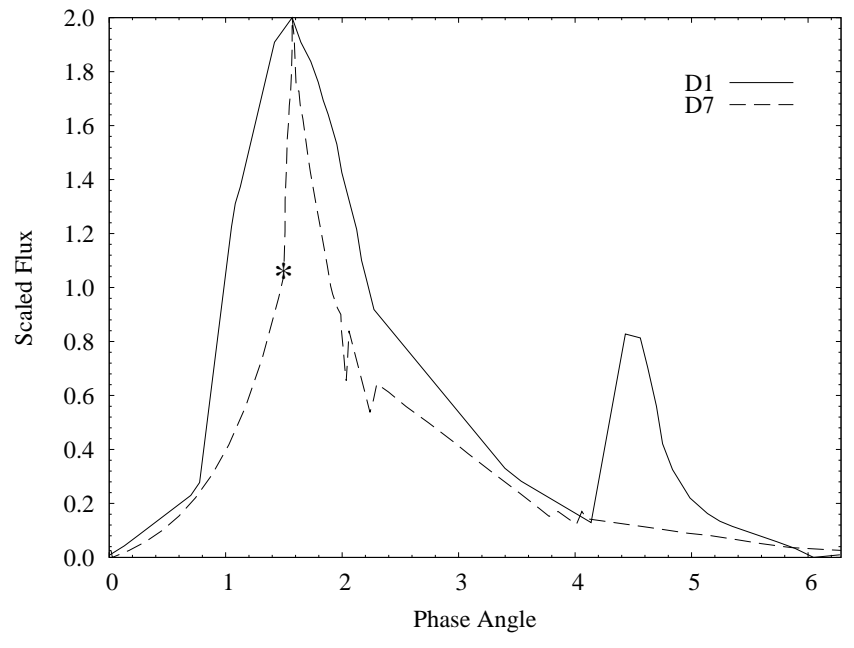

Figure 1. Driving functions $D_{1}$ and $D_{7}$. Both functions are scaled to unit amplitude and the maximum placed at $\pi / 2$ radians. The symbol '*' in $D 7$ marks the start of the observational data. Points to the left of this marker follow an exponential added by the present authors.

ted. When a fit has been established, it is then straightforward to recover the complete maser light curve at that $x y$ position.

The observational data that we use as driving functions in this work are, firstly, the NEOWISE data from G107.298+5.63 (Stecklum et al. 2018). The digitized form of this IR light curve is referred to as function $D 1$ in Paper 1, and we keep this nomenclature here. Secondly, we use the IR light curve in the $\mathrm{K}_{s}$ band from S255-NIRS3 (Uchiyama et al. 2020). This light curve is incomplete at early times, and we have completed it by fitting an exponential function that links the earliest epoch in Figure 3 of Uchivama et al. (2020) with the first observational data point. This latter function was not used quantitively in Paper 1, but is named $D 7$ here to follow on from the highest numbered function in Paper 1. Both driving functions are plotted in a normalized form in Fig. 1 with maximum light at a phase angle of $\pi / 2$. Digitization tasks were carried out using the WebPlotDigitizer tool, by Ankit Rohatg 1 .

\section{ANALYSIS}

We consider initially IR pumping. In this case, a change in optical depth parameter, as a proxy for the unsaturated maser inversion, follows the driving function. We use function $D 1$ for the G107.298+5.63 flare and function $D 7$ for S255-NIRS3. In our analysis of G107.298+5.63, the principal difference between the present work and our preliminary analysis in Paper 1 is that we now use $D 1$ to construct the fitting plot, rather than a sinusoid, so that our new plot in Fig. 2 is source-specific. As in Paper 1 we use, for the parameters of the maser response in the $-7.4 \mathrm{~km} \mathrm{~s}^{-1}$ feature, a duty cycle of 0.143 and the quoted variability index of 120 (Szymczak et al. 2016). We then read off from Fig. 2 the intercept of the orange contours at 0.143 and $\log _{10}(120)=2.08$ to recover the original optical depth parameter of the maser and the change in depth needed to generate the flare. The result is $\left(\tau_{\min }, \Delta \tau\right)=(5.14,3.15)$, marked with the $\times$ symbol in Fig. 2 and these figures correspond to an oscillation between conditions of weak and moderate saturation. Of course, there are significant

\footnotetext{
1 https://automeris.io/WebPlotDigitizer
}

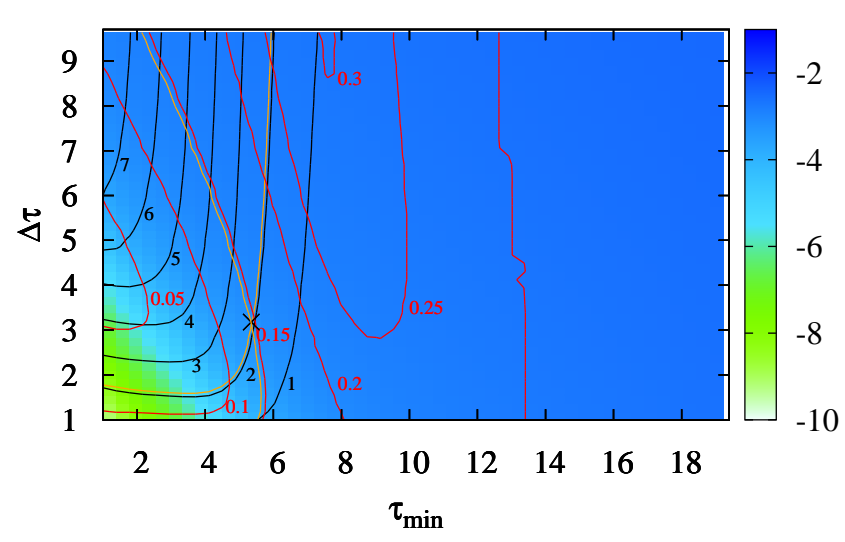

Figure 2. The maximum flux density achieved (represented by the colour scale), the base-10 logarithm of the variability index (black contours) and duty cycle of the maser (red contours) as functions of the maser optical depth parameter at minimum light ( $x$ axis) and the change in this parameter in moving from minimum to flare maximum ( $y$ axis). One contour from each set is plotted in orange for the observationally derived variability index and duty cycle, with the intercept marked by a black $\times$ symbol. This figure was constructed using the driving function D1, making it specific to G107.298+5.63.

modelling uncertainties attached to this fit, and to those calculated similarly below. We estimate the size of these uncertainties in Section 4 The third parameter, the maximum flux density achieved, is an additional constraint. At the intercept point given above, the scaled flux density has a value of $f_{\nu}=6.23 \times 10^{-4}$ from the colour scale in Fig. 2 With an observational flux density in Jy, and a distance to the source, we may calculate the required size of the maser source from eq.(A6) of Paper 1. Inserting our dimensionless flux density, the observational counterpart of $57.7 \mathrm{Jy}$, a loss rate in the $6.7-\mathrm{GHz}$ transition of $0.79 \mathrm{~Hz}$, as in Paper 1, and a source distance of $0.76 \mathrm{kpc}$ (Hirota et al. 2008), the model and measured flux densities are consistent if the maser object has a size of about 4.4 AU. We show the observed maser light curve and the light curve calculated at our fit position in the upper panel of Fig. 5. The derived size of the maser object is consitent with $E V N$ observations, provided that the sizes of individual maser objects are significantly smaller than an observed region 30-80 AU in extent (Szymczak et al. 2016), that contains several such objects.

In S255-NIRS3, there are two spectral features that might be considered problematic for analysis in view of their large flux density and/or variability index. We consider first the feature at $5.83 \mathrm{~km} \mathrm{~s}^{-1}$. For this feature, the maser flux density is above half the peak value for 45 days out of the 1100 flaring days, corresponding to a duty cycle of 0.041 , and its variability index is quoted as 27 (Uchiyama et al. 2020). We attempt to fit these parameters with a new plot, Fig. 3, that differs from Fig. 2] in being prepared with the IR driving function $D 7$, instead of $D 1$, making Fig. 3 specific to S255-NIRS3. Using the same fitting procedure applied above, we find that the 0.041 orange contour meets its variability index counterpart, delineating $\log _{10}(27)=1.43$, at $\left(\tau_{\min }, \Delta \tau\right)=(5.72,2.19)$. The dimensionless flux density at this point is $f_{\nu}=4.22 \times 10^{-4}$. To make this consistent with an observed peak flux density of $1632 \mathrm{Jy}$, with S255-NIRS3 at a distance of $1.78 \mathrm{kpc}$ (Burns et al. 2016), requires a maser object with a size of $67 \mathrm{AU}$. We show the observed maser light curve and our light curve at the fitting point in the middle panel of Fig.5

The maser feature at $6.42 \mathrm{~km} \mathrm{~s}^{-1}$ in S255-NIRS3 has no fit point in Fig. 3. its duty cycle of 0.39 is too large to be consis- 


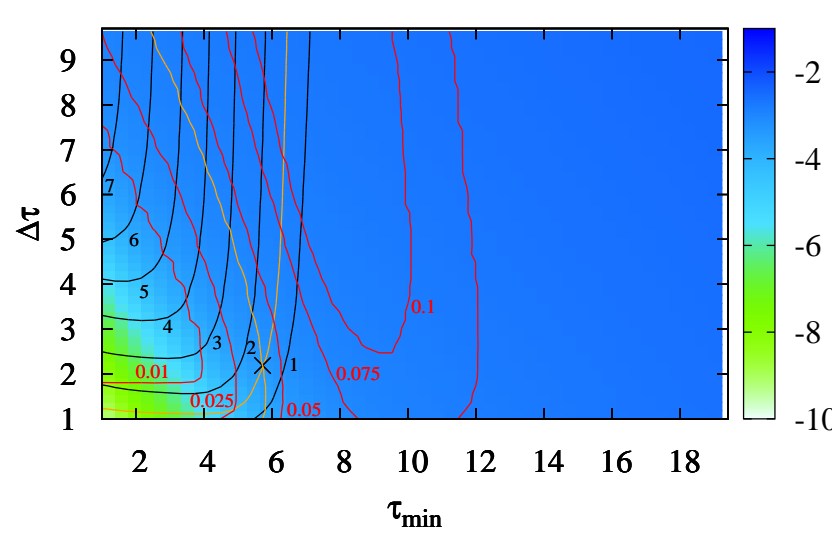

Figure 3. As for Fig. 2 but prepared with the $D 7$ driving function, specific to the S255-NIRS3 source. The black cross marks the variable pumping fit position for the $-5.83 \mathrm{~km} \mathrm{~s}^{-1}$ feature.

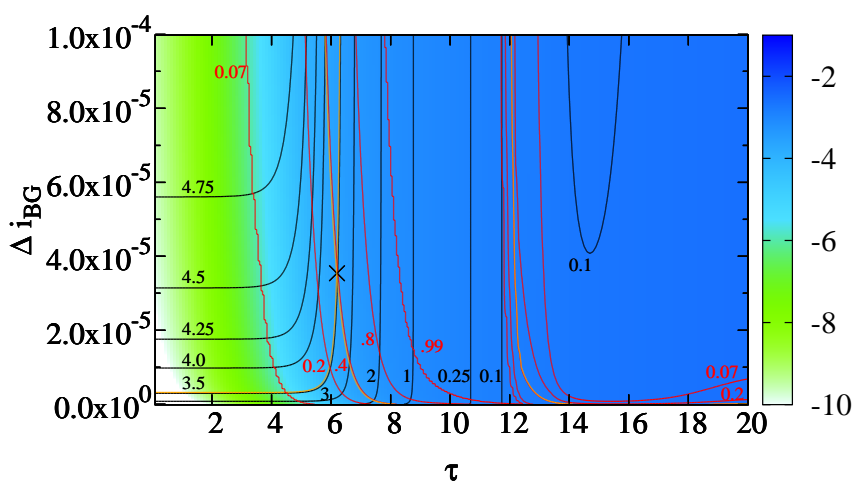

Figure 4. As for Fig. 2 but prepared with $D 7$ driving function, and the $y$ axis variable is now the change in the intensity of background radiation. Use of the $D 7$ function makes the plot specific to the S255-NIRS3 source. The cross marks the fit position for the $-6.42 \mathrm{~km} \mathrm{~s}^{-1}$ feature.

tent with its variability index of 3400 (the base 10 logarithm of this number is 3.531). On these grounds, we reject an IR pumping mechanism for this component and seek instead a fit to a variable background mechanism. There is an immediate problem with this approach because the $D 7$ driving function is based on IR observations in the $\mathrm{K}_{s}$ band, not on the radio continuum background at $6.7 \mathrm{GHz}$. However, in the absence of any contemporaneous radio background data, we still use the $D 7$ driving function to prepare Fig. 4 in the knowledge that this functional form could be quite wrong. The $y$ axis in Fig. 4 is now the change in the intensity of the background radiation; the optical depth parameter, once chosen, is now a constant during the flare. With $D 7$ as the driving function, it becomes possible to find a fit for the $6.42 \mathrm{~km} \mathrm{~s}^{-1}$ feature. The intercept of the 0.39 duty-cycle contour and the $\log _{10}(3400)=3.53$ variability contour (both orange in Fig. (4) occurs at $\left(\tau, \Delta i_{B G}\right)=\left(6.20,3.56 \times 10^{-5}\right)$, noting that $i_{B G}$ is measured in units of the saturation intensity of the maser transition.

The scaled flux density at the position of the fit in Fig. 4 is $2.432 \times 10^{-4}$, and this figure, to be consistent with an observed flux density of $1705 \mathrm{Jy}$ (see Table 1 of Uchiyama et al. 2020), requires a cloud size of $89.6 \mathrm{AU}$. This is a similar size to that required for the feature at $5.83 \mathrm{~km} \mathrm{~s}^{-1}$, even though the pumping mechanisms are probably different, and is a consequence of the similar observed peak flux densities and model flux densities achieved for

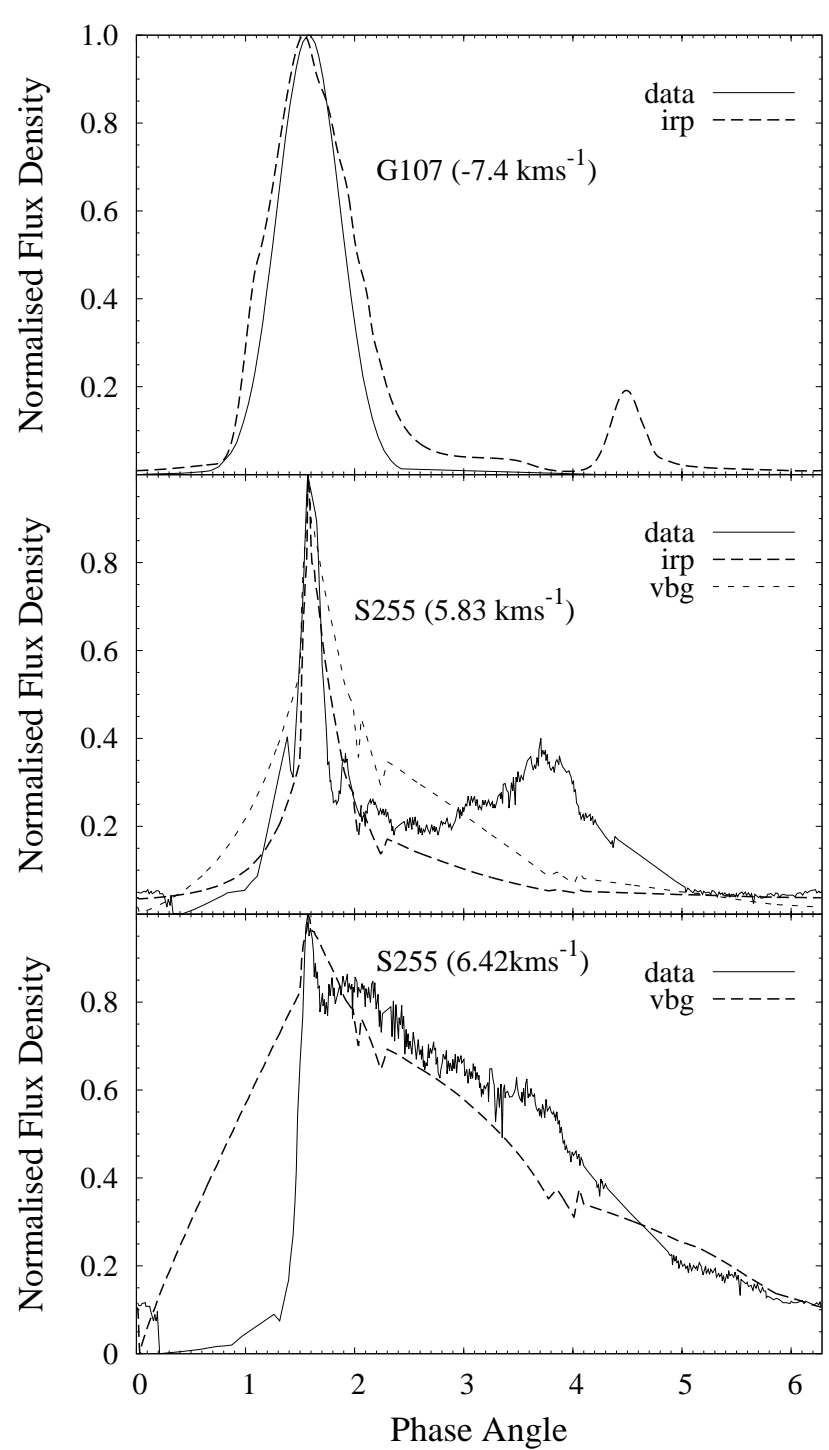

Figure 5. Our fits based on two model parameters compared with observational maser flare data for the three maser light curves analysed. Fits using the IR pumping mechanism are marked 'irp'; those using the variable background are marked 'vbg'. Observational data for the S255-NIRS3 features (middle and bottom panels) were digitized from graphs in Uchivama et al. (2020). Data for G107.298+5.63 (top panel) follow the best-fit gaussian in Fuijsawa et al. (2014), since no light curve is plotted for the $-7.4 \mathrm{~km} \mathrm{~s}^{-1}$ feature in Szymczak et al. (2016), and only a copy of the best-fit gaussian is plotted in Stecklum et al. (2018). In all cases, the functions are plotted over a phase-angle range of $0-2 \pi$ with the flux densities normalised to the values at maximum light. This amounts to choosing the cloud size that makes the model flux density agree exactly with the observed value in Jy (see text above). Times corresponding to a phase angle of $2 \pi$ are $34.6 \mathrm{~d}$ (the maser period) in the top panel and $1100 \mathrm{~d}$ in the other two panels.

the two features. The maser light curve and our model counterpart are shown in the bottom panel of Fig. 5 The required cloud size is probably consistent with results from VLBI observations: Observations of S255-NIRS3 with the EVN during the burst resolved out over 90 per cent of the single-dish flux density with a beam of $3 \times 5$ milliarcsec (Szymczak et al. 2018). At $1.78 \mathrm{kpc}$, this implies that most of the maser emission arises from a structure, or structures, significantly larger than 7 AU. Lower resolution JVLA observations suggest the maser structures could be up to $430 \mathrm{AU}$ in size (Moscadelli et al. 2017). Our values of 60-90 AU, required 
for consistency between model and observational flux densities in S255-NIRS3, therefore fall neatly into the range of scales associated with the majority of the maser flux density.

If there is a requirement that the same (variable background) mechanism should be used for the $5.83 \mathrm{~km} \mathrm{~s}^{-1}$ feature, then an approximate fit can be made from Fig. 4 In this case we demand a model flux density $\left(1.02 \times 10^{-5}\right)$ corresponding to the possible 430 AU structures in S255-NIRS3, but compromise on the duty cycle $(0.080)$ and variability index (624) that are found at $\left(\tau, \Delta i_{B G}\right)=\left(5.7,7.0 \times 10^{-7}\right)$. The fit with these parameters is shown as the curve marked 'vbg' in Fig. 5 .

\section{DISCUSSION}

We have demonstrated that our global model of radiation-driven maser flares can be used to fit a variety of maser light curves, including 'giant flares', with peak flux densities in excess of $1000 \mathrm{Jy}$ for sources at distances of order $1 \mathrm{kpc}$. No novel mechanisms, such as, for example, superradiant emission (Rajabi \& Houde 2017) are necessarily required. The variability index and duty cycle parameters usually exclude one or other of the IR pumping and variable background mechanisms. We have modelled the $5.83 \mathrm{~km} \mathrm{~s}^{-1}$ feature with both mechanisms, but only the IR pumping fit can accommodate all the observational constraints. The fitting procedure is quite simple, involving only three observational parameters of the maser flare that are used to recover three model variables related to the amplification and saturation of the maser. The 3D nature of the model allows for a reasonably strong consistency check by calculating the required size of the maser object needed to equate the observed and modelled flux densities.

We have attempted to estimate uncertainties in our fits by calclating additional contour intercepts for a different model in which the unsaturated inversions rise as the square of the distance from the centre of the model cloud. We refer to this as the shell distribution. This distribution is arguably a better representation of the unsaturated inversion than a uniform model in the case of pumping by an optically thick IR transition. In the same order as presented above, the alternative fits are $\left(\tau_{\min }, \Delta \tau\right)=(4.65,3.91)$ with a flux density of $1.1 \times 10^{-3}$ for G107.298+5.63, and $\left(\tau_{\min }, \Delta \tau\right)=$ $(5.00,2.75)$ with a flux density of $5.7 \times 10^{-4}$ for the $5.83 \mathrm{~km} \mathrm{~s}^{-1}$ feature of S255-NIRS3. No fit could be found for the variable background model and shell profile in the case of the $6.42 \mathrm{~km} \mathrm{~s}^{-1}$ feature. The closest approach if the correct duty cycle is required is $\left(\tau, \Delta i_{B G}\right)=\left(5.0,8 \times 10^{-5}\right)$, where the variability index is 2150 . However, in the case of a variable background, the reason for the adoption of the shell distribution of unsaturated inversion no longer applies.

Our estimation of uncertainties suggests that $\tau_{\min }$, or $\tau$ for the background model, is uncertain by approximately \pm 1 . For the IR pumped fits, the uncertainty in $\Delta \tau=0.66$ averaged over both fits. The change in background is considerably more uncertain as no fit was found for the shell model, and because of the almost vertical contours close to the fit position in Fig. 4 Maser parameters recovered from the analysis show that, in all three cases modelled here, the pre-flare, or minimum light, maser objects have only modest saturation, with a narrow range of 5.14-6.20 in optical depth parameter within the uniform model. An uncertainty of \pm 1 in $\tau_{\min }$ does not change this conclusion. At flare maximum, flux densities achieved by the flaring objects are significant, but still a factor of 5-10 lower than those corresponding to a strongly-saturated maser. Flux densities derived from the shell-model fits are approximately a factor of two larger than for the uniform model, dividing required cloud sizes by a factor of $\sim \sqrt{2}$.

A glance at Fig. 5 suggests that the exponential used to fill in the missing IR data at early times in S255-NIRS3 is not particularly good, and that the real function is something even steeper. We note that our model cannot reproduce secondary peaks, such as that visible in the middle panel of Fig. 5unless such a peak is also present in the driving function. The use of the $D 7$ IR function in the case of the variable background fit is highly speculative, but a fit could be obtained for the uniform cloud model. It should be noted that the difference in background over the flare is important, rather than the ratio, so results are dominated by the largest background levels used: a background range of $10^{-6}-10^{-4}$ would therefore produce an almost identical Fig. 4 to the one plotted, which uses $10^{-9}-10^{-4}$. For a frequency in the Rayleigh-Jeans limit, and parameters for the 6.7-GHz transition of methanol from Paper 1, the background intensity at the fit position in Fig. 4 corresponds to bathing the maser cloud in unattenuated, and geometrically undiluted, black-body radiation at a temperature of $3080 \mathrm{~K}$.

Future models could include a core-halo density structure, allowing us to address the possible presence of objects with scales of order 1-500 AU and the issue of missing flux in VLBA observations when compared with single-dish spectra.

\section{ACKNOWLEDGMENTS}

MDG and SE acknowledge funding from the UK Science and Technology Facilities Council (STFC) as part of the consolidated grant ST/P000649/1 to the Jodrell Bank Centre for Astrophysics at the University of Manchester. MDG acknowledges financial support from the National Astronomical Research Institute of Thailand (NARIT) whilst on sabbatical at their HQ in Chiang Mai, Thailand. This work was performed, in part, using the DiRAC Data Intensive service at Leicester, operated by the University of Leicester IT Services, which forms part of the STFC DiRAC HPC Facility (www.dirac.ac.uk). The equipment was funded by BEIS capital funding via STFC capital grants ST/K000373/1 and ST/R002363/1 and STFC DiRAC Operations grant ST/R001014/1. DiRAC is part of the National e-Infrastructure. Data used in this work was generated under DiRAC award dp124.

\section{DATA AVAILABILITY STATEMENT}

The data underlying this article will be shared on reasonable request to the corresponding author.

\section{REFERENCES}

Burns R. A., Handa T., Nagayama T., Sunada K., Omodaka T., 2016, MNRAS, 460, 283

Fujisawa K., Takase G., Kimura S., Aoki N., Nagadomi Y., Shimomura T., Sugiyama K., Motogi K., Niinuma K., Hirota T., 2014, PASJ, 66, 78

Gray M. D., Baggott J., Westlake J., Etoka S., 2019, MNRAS, 486, 4216

Gray M. D., Etoka S., Travis A., Pimpanuwat B., 2020, MNRAS, 493, 2472 (Paper 1)

Gray M. D., Mason L., Etoka S., 2018, MNRAS, 477, 2628 
Hirota T., Ando K., Bushimata T., Choi Y. K., Honma M., Imai H., Iwadate K., Jike T., Kameno S., Kameya e. a., 2008, PASJ, 60, 961

Moscadelli L., Sanna A., Goddi C., Walmsley M. C., Cesaroni R., Caratti o Garatti A., Stecklum B., Menten K. M., Kraus A., 2017, A\&A, 600, L8

Rajabi F., Houde M., 2017, Science Advances, 3, e1601858

Stecklum B., Caratti o Garatti A., Hodapp K., Linz H., Moscadelli L., Sanna A., 2018, in Tarchi A., Reid M. J., Castangia P., eds, Astrophysical Masers: Unlocking the Mysteries of the Universe Vol. 336 of IAU Symposium, Infrared variability, maser activity, and accretion of massive young stellar objects. pp 37-40

Szymczak M., Olech M., Wolak P., Bartkiewicz A., Gawroński M., 2016, MNRAS, 459, L56

Szymczak M., Olech M., Wolak P., Gérard E., Bartkiewicz A., 2018, A\&A, 617, A80

Uchiyama M., Yamashita T., Sugiyama K., Nakaoka T., Kawabata M., Itoh R., Yamanaka M., Akitaya H., Kawabata K., Yonekura Y., Saito Y., Motogi K., Fujisawa K., 2020, PASJ, 72, 4 